\title{
NUMERICAL ASSESSMENT OF THE MEAN POWER PRODUCTION OF A COMBINED WIND AND WAVE ENERGY PLATFORM
}

\author{
Thomas Soulard \\ LUNAM Université \\ École Centrale de Nantes \\ LHEEA - UMR CNRS 6598 \\ Nantes, FRANCE
}

\author{
Aurélien Babarit \\ LUNAM Université \\ École Centrale de Nantes \\ LHEEA - UMR CNRS 6598 \\ Nantes, FRANCE
}

\begin{abstract}
This paper synthesizes the performance evaluation of a hybrid ocean wave energy converter. The case of interest represents a singular approach to combine a $5 \mathrm{MW}$ wind turbine with floating oscillating wave surge converters (OWSCs). The first section describes the comprehensive set of equations of motion in both frequency and time domain. The mathematical and hydrodynamic assumptions are highlighted together with the numerical model. The second part starts with the assessment of the initial performance of this device, carried out on in-house simulation codes. The first stage of numerical validation is based on the linear transfer functions (RAOs) results. The analysis of these RAOs also exposes interesting mechanical properties of this particular device. Eventually, the annual average absorbed power figures are extracted from the power matrices calculated for a few different sites.
\end{abstract}

Keywords - Wave energy converter, floating wind turbine, combined energy platform.

\section{INTRODUCTION}

The MARINA Platform project is a European initiative created to bring expertise from offshore wind industries together with ocean energy specialists, in order to reduce costs for deep water offshore platforms. Additional information is available on the project website [1]. One of the major goals is to create a pragmatic approach to evaluate different hybrid platforms designed by a consortium of experts. Basic cost evaluations were carried out among the partners to select a few promising concepts. One of the methods chosen to establish a ranking for the different types of devices was based on the capture width classification proposed in [2]. However, more detailed analyses are required in order to compare the performance of the few selected best candidates. The aim of this paper is to describe the process for one particular concept combining wind and wave powers.

The first section of this paper will focus on the system description, starting with a brief presentation of the mechanical parameters of this platform. The comprehensive set of equations required to build the Wave to Wire (W2W) model is then detailed. The methodology relies on numerical modeling and has already been proven in [4].

In the second part, the initial performance of the platform is assessed based on frequency domain simulations. A brief set of numerical tests have been carried out in order to validate the different models, in both frequency and time domain. Finally, the power matrix is displayed together with the mean annual power absorption at five different representative wave sites along the European coasts.

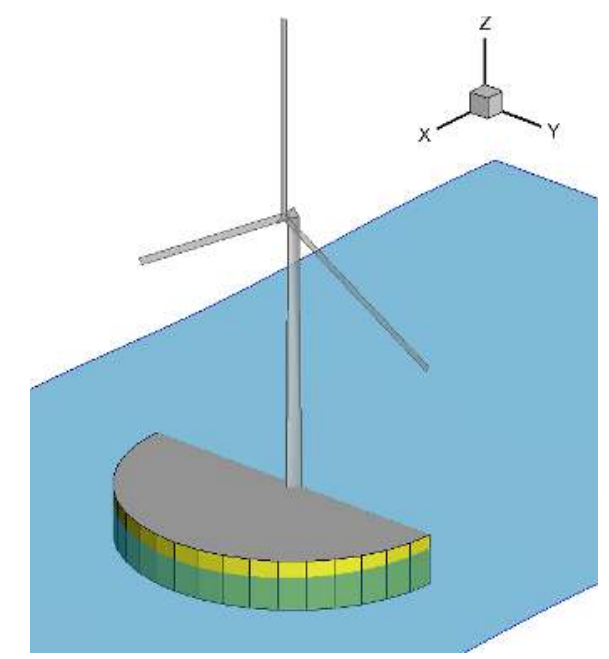

Figure 1: The combined platform considered. 


\section{1 - SYSTEM DESCRIPTION}

The platform considered in this study is a combined wind and wave energy device. It is composed of a semicircular base on which stands a $5 \mathrm{MW}$ horizontal axis wind turbine. On the wave facing side of the platform, 20 wave energy converters (WECs) of the surging type are attached. The relative motion between the platform and the WECs is used to produce energy. The sea clam device [3] was mentioned during the creation process.

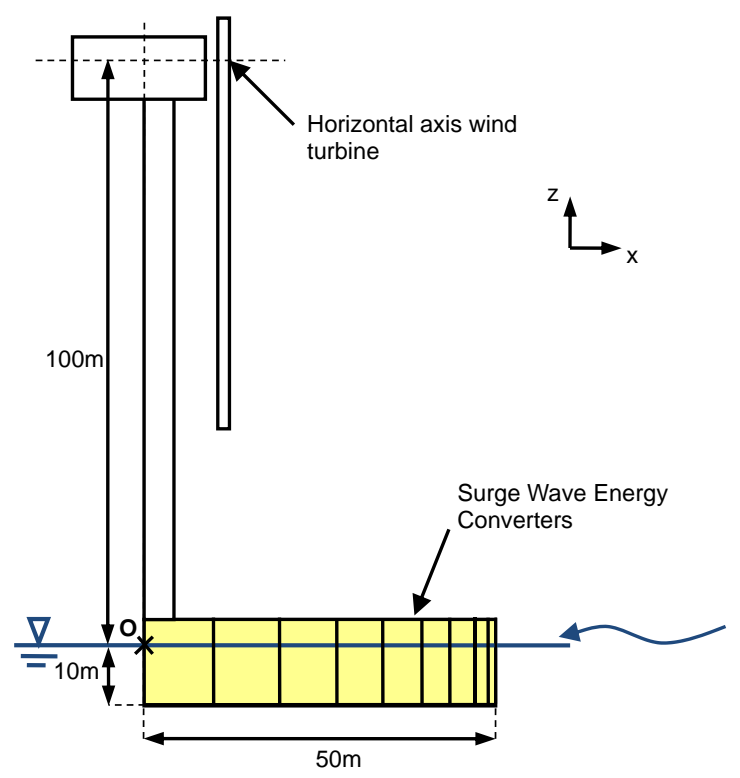

Figure 2: Dimension of the Platform.

A 3D picture of the system is shown in Figure 1, whereas Figure 2 is a side view highlighting the main dimensions of the platform. Its radius is $50 \mathrm{~m}$, the draft is $10 \mathrm{~m}$. The power rating of each WEC is expected to be $250 \mathrm{~kW}$. As a result, the total rated power of the wave energy part of the platform is $5 \mathrm{MW}$, and the rated power of the device is 10MW. Contribution from wind and wave to the total energy production of the platform would be balanced. For this specific design, each WEC is excited one after the other when the wave travels along the machine. Then, the primary power production is expected to be smoother than with point absorbers or barrier type wave energy converters. Therefore, the need for energy storage could be reduced.

\section{1 - Dimensions and mechanical parameters}

Dimensions of the platform are shown in Figure 2, along with the location of the origin of the coordinate system (marked by $\mathrm{O}$ ). A more comprehensive list of mechanical properties is displayed in Table 1. The WT design was based on the NREL $5 \mathrm{MW}$ wind turbine model. Its mechanical characteristics where taken from [6].

\begin{tabular}{|c|c|c|c|}
\hline Property & Notation & Value & Unit \\
\hline \multicolumn{4}{|l|}{ Platform } \\
\hline Radius & $\mathrm{R}$ & 50 & $\mathrm{~m}$ \\
\hline Height & $\mathrm{H}$ & 15 & $\mathrm{~m}$ \\
\hline Draft & $\mathrm{D}$ & 10 & $\mathrm{~m}$ \\
\hline Breadth & B & 10 & $\mathrm{~m}$ \\
\hline Displacement & V & 39000 & $\mathrm{t}$ \\
\hline Wetted surface & $\mathrm{S}_{\mathrm{p}}$ & 6500 & $\mathrm{~m}^{2}$ \\
\hline Significant Surface & $\mathrm{S}_{\text {tot }}$ & 11710 & $\mathrm{~m}^{2}$ \\
\hline Waterplane area & $\mathrm{S}_{\mathrm{wp}}$ & 3900 & $\mathrm{~m}^{2}$ \\
\hline $\mathrm{x}$ - position of CoG & $\mathrm{x}_{\mathrm{Gp}}$ & 21.2 & $\mathrm{~m}$ \\
\hline$z$ - position of CoG & $\mathrm{Z}_{\mathrm{Gp}}$ & -2.5 & $\mathrm{~m}$ \\
\hline Moment of inertia along y-axis & $\mathrm{I}_{\mathrm{yyp}}$ & 11600000 & t. $\mathrm{m}^{2}$ \\
\hline \multicolumn{4}{|l|}{ WEC } \\
\hline Number of panels & $\mathrm{N}$ & 20 & - \\
\hline Width & W & 8 & $\mathrm{~m}$ \\
\hline Wetted surface & $\mathrm{S}_{0}$ & 120 & $\mathrm{~m}^{2}$ \\
\hline Mass & $\mathrm{m}_{\mathrm{i}}$ & 36 & $\mathrm{t}$ \\
\hline $\mathrm{z}$ - position of CoG & $\mathrm{z}_{\mathrm{Gi}}$ & -2.5 & $\mathrm{~m}$ \\
\hline Moment of inertia along y axis & $\mathrm{I}_{\text {yyi }}$ & 2600 & t. $m^{2}$ \\
\hline \multicolumn{4}{|l|}{ Wind Turbine } \\
\hline Rotor diameter & $\mathrm{d}_{\text {rotor }}$ & 126 & $\mathrm{~m}$ \\
\hline Total mass & $\mathrm{M}_{\mathrm{WT}}$ & 697 & $\mathrm{t}$ \\
\hline Rotor mass & $\mathrm{M}_{\text {rotor }}$ & 110 & $\mathrm{t}$ \\
\hline Nacelle mass & $\mathrm{M}_{\text {nacelle }}$ & 240 & $\mathrm{t}$ \\
\hline Tower mass & $\mathrm{M}_{\text {tower }}$ & 347 & $\mathrm{t}$ \\
\hline Nacelle height & $z_{N}$ & 100 & $\mathrm{~m}$ \\
\hline $\mathrm{z}$ - position of $\mathrm{CoG}$ & $z_{W T}$ & 64 & $\mathrm{~m}$ \\
\hline
\end{tabular}

Table 1: Dimensions and mechanical parameters of the system.

\section{2 - PTO and Control}

A linear Power Take Off (PTO) scenario was modeled; with a damping coefficient denoted $B_{P T O}$ and a spring coefficient $K_{\text {PTO }}$. Only positive values for $K_{P T O}$ were considered, because this configuration can be achieved with physical springs.

Damping and conversion of the mechanical energy could be achieved using hydraulic cylinders or linear generators. The choice should be made based on considerations of cost, maintenance, availability, etc. which is out of the scope of this study.

\section{3 - Site and wave resource}

In order to estimate the annual energy absorption, 5 sites located on the Atlantic coast of Europe were selected. With mean annual wave resources varying from 15 to $80 \mathrm{~kW} / \mathrm{m}$, they are thought to be representative. As an example, the wave scatter diagram for the French site Yeu is displayed below, in percentage of occurrences. 


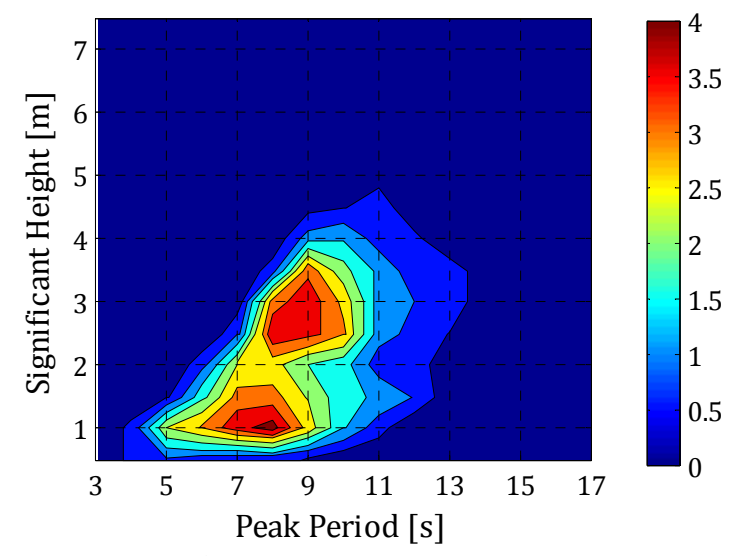

Figure 3: Yeu Scatter diagram, in percentage of occurrences. The annual power input is $26 \mathrm{~kW} / \mathrm{m}$.

\section{2- EQUATION OF MOTION}

\section{1 - Assumptions and notations}

Waves are assumed to be monodirectional and propagating in the $\mathrm{x}$ direction. Let $\mathrm{G}_{0}$ be the gravity center of the platform, $\mathrm{x}_{0}$ and $\mathrm{z}_{0}$ being its surge and heave motions. Let $\theta_{0}$ be the pitch angle of the whole system. The variable $r_{i}$ will refer to the relative excursion of the WEC number $i$ and $\mathrm{G}_{\mathrm{i}}$ is its gravity center. The angular location of each flap will be noted $\alpha_{i} \in$ $\left[-\frac{\pi}{2} ; \frac{\pi}{2}\right]$. C will refer to the center of the circular part of the platform. Its vertical position is taken in the same horizontal plane as the gravity center of the platform. In a first approach, the origin of the fixed Cartesian frame, $\mathrm{O}$, is set equal to $\mathrm{C}$ at rest.

It is assumed that all motions are of small amplitude. Thus, the second order effects are neglected. For convenience, a generalised motion vector $\mathrm{X}_{\mathrm{i}, \mathrm{i}=0, \mathrm{~N})}$ was introduced for each body. It is expressed at $G_{0}$, and has 6 rows: surge $\mathrm{x}_{\mathrm{i}}$, sway $\mathrm{y}_{\mathrm{i}}$, heave $z_{i}$, roll $\varphi_{i}$, pitch $\theta_{i}$ and yaw $\psi_{i}$

\section{2 - Kinematics}

The absolute position of $G_{i}$ is a function of $\alpha_{i}$ :

$$
\begin{gathered}
\overrightarrow{O G_{\imath}}=\overrightarrow{O G_{0}}+\overrightarrow{G_{0} C}+\overrightarrow{C G_{\imath}} \\
\overrightarrow{O G_{\iota}}=\left(\begin{array}{c}
x_{0} \\
0 \\
z_{0}
\end{array}\right)^{0}+\left(\begin{array}{c}
-r_{0} \cos \theta_{0} \\
0 \\
r_{0} \sin \theta_{0}
\end{array}\right)^{0}+\left(\begin{array}{c}
\left(R+r_{i}\right) \cos \alpha_{i} \cos \theta_{0} \\
\left(R+r_{i}\right) \sin \alpha_{i} \\
-\left(R+r_{i}\right) \cos \alpha_{i} \sin \theta_{0}
\end{array}\right)^{0}
\end{gathered}
$$

Its velocity is:

$$
\vec{V}_{0}\left(G_{i}\right)=\left(\begin{array}{c}
u+r_{0} \theta_{0} \sin \theta_{0}-\left(R+r_{i}\right) \cos \alpha_{i} \theta_{0} \sin \theta_{0}+r_{l} \cos \alpha_{i} \cos \theta_{0} \\
r_{l} \sin \alpha_{i} \\
w+r_{0} \theta_{0} \cos \theta_{0}-\left(R+r_{i}\right) \cos \alpha_{i} \theta_{0} \cos \theta_{0}+r_{l} \cos \alpha_{i} \sin \theta_{0}
\end{array}\right)^{0}
$$

Based on the small angle approximation:

$$
\vec{V}_{0}\left(G_{i}\right)=\left(\begin{array}{c}
u+r_{l} \cos \alpha_{i} \\
r_{l} \sin \alpha_{i} \\
w+\left(r_{0}-R \cos \alpha_{i}\right) \theta_{0}
\end{array}\right)^{0}
$$

The acceleration and the dynamic moment of the WECs can be deduced from Eq.(4).

\section{3 - Forces}

\subsubsection{Wave excitation and radiation force}

The system is fully defined with 21 bodies, which are assumed to be independent. According to the linear potential flow theory, the generalized wave excitation and radiation forces (for the flap $i$ ) can be written:

$$
\boldsymbol{F}_{e x, i}\left(G_{0}\right)+\boldsymbol{F}_{r a d, i}\left(G_{0}\right)=\boldsymbol{F}_{e x, i}-\sum_{j=0}^{N} \boldsymbol{C} \boldsymbol{M}_{i j} \boldsymbol{X}_{\boldsymbol{j}}-\sum_{j=0}^{N} \boldsymbol{C} \boldsymbol{A}_{i j} \boldsymbol{X}_{\boldsymbol{j}}
$$

In which $X_{j}$ are expressed at $G_{0}$.

Let's consider now $\boldsymbol{X}=\left(\begin{array}{llllll}x_{0} & z_{0} & \theta_{0} & r_{1} & \cdots & r_{N}\end{array}\right)^{T}$, the actual motion vector of the complete platform.

The numbering of flaps is directly related to the degrees of freedom number (DoF). Indeed, the first three DoFs are allocated to the platform motion $\left(\begin{array}{lll}x_{0} & z_{0} & \theta_{0}\end{array}\right)$ whereas the first flap on the side of the platform will be called "panel 4 ". Then, a flap on the front of the platform, facing the waves, is referred as “panel 13”.

Using Eq.(1) and reminding that the $\boldsymbol{X}_{j}$ are expressed at $G_{0}$, one can show:

$$
\boldsymbol{X}_{i}=\left(\begin{array}{c}
R \cos \alpha_{i}-r_{0} \\
0 \\
0 \\
0 \\
0 \\
0
\end{array}\right)+\boldsymbol{T}_{i} \boldsymbol{X}
$$

With

$\boldsymbol{T}_{i}=\left(\begin{array}{cccccc}1 & 0 & 0 & \cdots & \cos \alpha_{\mathrm{i}} & \cdots \\ 0 & 0 & 0 & \cdots & \sin \alpha_{\mathrm{i}} & \cdots \\ 0 & 1 & 0 & \cdots & 0 & \cdots \\ 0 & 0 & 0 & \cdots & 0 & \cdots \\ 0 & 0 & 1 & \cdots & 0 & \cdots \\ 0 & 0 & 0 & \cdots & 0 & \cdots\end{array}\right)$

After defining the assembled matrix $\boldsymbol{T}=\left(\boldsymbol{T}_{\mathbf{0}} \cdot \cdots \boldsymbol{T}_{N}\right)^{T}$, one can show:

With

$$
F_{e x, i}\left(G_{0}\right)+F_{r a d, i}\left(G_{0}\right)=F_{e x, i}-C M_{i} T X-C A_{i} T X
$$

$C M_{i}=\sum_{j} C M_{i j} T_{j}$

$C A_{i}=\sum_{j} C A_{i j} T_{j}$

\subsubsection{Hydrostatic force:}

\section{- WECs}

The hydrostatic force is calculated for each flap independently. The coordinates $(\xi, v)$ will refer to a point $\mathrm{M}$ of wetted surface 
of the flap $i$, when $r_{i}=0$. This surface shall be noted as $S_{i, 0}$. One can show that the coordinates of $\mathrm{M}$ in the reference frame of the platform are:

$$
\overrightarrow{G_{O} M}=\left(\begin{array}{c}
-r_{0}+\left(R+r_{i}\right) \cos \alpha_{i}-\xi \sin \alpha_{i} \\
\left(R+r_{i}\right) \sin \alpha_{i}+\xi \cos \alpha_{i} \\
v+d_{i}
\end{array}\right)^{b}
$$

With $d_{i}=\overrightarrow{G_{O} B_{l}} \cdot \vec{z}, B_{i}$ being the buoyancy center of the flap $i$. In the reference frame and after linearization:

$$
\overrightarrow{G_{O} M}=\left(\begin{array}{c}
-r_{0}+\left(R+r_{i}\right) \cos \alpha_{i}-\xi \sin \alpha_{i}+\left(v+d_{i}\right) \theta_{0} \\
\left(R+r_{i}\right) \sin \alpha_{i}+\xi \cos \alpha_{i} \\
-\left(-r_{0}+R \cos \alpha_{i}-\xi \sin \alpha_{i}\right) \theta_{0}+v+d_{i}
\end{array}\right)^{0}
$$

Eventually, the submergence of the point $\mathrm{M}$ is given by

$$
z=z_{0}-\left(-r_{0}+R \cos \alpha_{i}-\xi \sin \alpha_{i}\right) \theta_{0}+v+d_{i}
$$

By definition the hydrostatic force is the integral of the pressure term $(\rho g z)$ on the wetted surface, it has the orientation of the normal $\vec{n}=\left(\cos \alpha_{i} \sin \alpha_{i}-\theta_{0} \cos \alpha_{i}\right)^{T, 0}$. Here, one must be careful about the actual volume displaced by the flap. If $r_{i} \neq 0$, the flap has a volume equal to $S_{i, 0} r_{i}$, which creates a vertical contribution in the hydrostatic force. Therefore, the actual total surface where the hydrostatic force applies can be expressed as $S_{i}=S_{i, 0} \cup S_{i, 0}{ }^{\prime}$ as described in Figure 4.

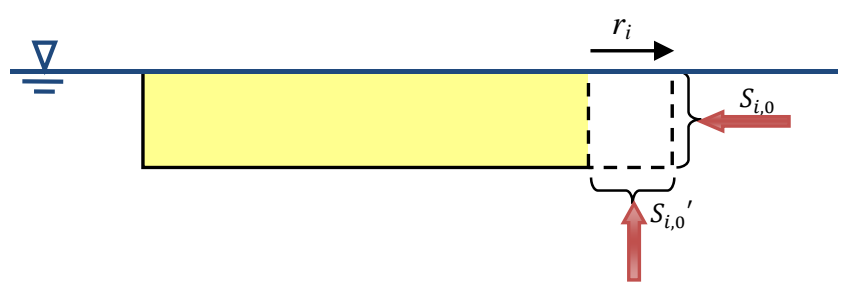

Figure 4: Sketch of the hydrostatic forces applying on a flap.

Therefore, the pressure has to be integrated also for a point $\mathrm{P}$, located on $S_{i, 0}{ }^{\prime}$ by its coordinate $(\tau, \kappa)$. After integration of each component, the total force can be obtained:

$$
\begin{aligned}
\overrightarrow{F_{H, l}}=\rho g d_{i} S_{0}\left(\begin{array}{c}
\cos \alpha_{i} \\
\sin \alpha_{i} \\
0
\end{array}\right)^{0}+\rho g z_{0} S_{0}\left(\begin{array}{c}
\cos \alpha_{i} \\
\sin \alpha_{i} \\
0
\end{array}\right)^{0} \\
\quad+\rho g \theta_{0} S_{0}\left(\begin{array}{c}
\left(r_{0}-R \cos \alpha_{i}\right) \cos \alpha_{i} \\
\left(r_{0}-R \cos \alpha_{i}\right) \sin \alpha_{i} \\
-d_{i} \cos \alpha_{i}
\end{array}\right)^{0}+\rho g S_{0}\left(\begin{array}{l}
0 \\
0 \\
r_{i}
\end{array}\right)^{0}
\end{aligned}
$$

And the moment force at $G_{0}$ is given by:

$$
\begin{aligned}
& \overrightarrow{\mathcal{M}_{H, l}}\left(G_{0}\right) \\
& =\left(\begin{array}{c}
R r_{i} \sin \alpha_{i}-d_{i}{ }^{2} \sin \alpha_{i}-z_{0} d_{i} \sin \alpha_{i}+\theta_{0} d_{i}\left(-2 r_{0}+R \cos \alpha_{i}\right) \sin \alpha_{i} \\
\left(r_{0}-R \cos \alpha_{i}\right) r_{i}+d_{i}{ }^{2} \cos \alpha_{i}+z_{0} d_{i} \cos \alpha_{i}+\theta_{0} d_{i}\left(r_{0}-R \cos \alpha_{i}\right) \cos \alpha_{i} \\
-r_{0} d_{i} \sin \alpha_{i}-r_{0} z_{0} \sin \alpha_{i}+\left(-r_{0}{ }^{2}+d_{i}{ }^{2}+r_{0} R \cos \alpha_{i}\right) \theta_{0} \sin \alpha_{i}
\end{array}\right)^{0}
\end{aligned}
$$

Finally, one can write the generalized hydrostatic force acting on each flap

$$
\boldsymbol{F}_{\boldsymbol{H}, \boldsymbol{i}}\left(G_{0}\right)=\boldsymbol{F}_{\boldsymbol{H}, \boldsymbol{i}}\left(G_{0}\right)-\boldsymbol{K}_{\boldsymbol{H}, \boldsymbol{i}} \boldsymbol{X}
$$

With

$$
\boldsymbol{F}_{H 0, i}\left(G_{0}\right)=\rho g d_{i} S_{0}\left(\begin{array}{c}
\cos \alpha_{i} \\
\sin \alpha_{i} \\
0 \\
-d_{i} \sin \alpha_{i} \\
d_{i} \cos \alpha_{i} \\
-r_{0} \sin \alpha_{i}
\end{array}\right)^{0}
$$

$\boldsymbol{K}_{H, i}$

$$
=-\rho g S_{0}\left(\begin{array}{cccccc}
0 & \cos \alpha_{i} & \left(r_{0}-R \cos \alpha_{i}\right) \cos \alpha_{i} & \cdots & 0 & \cdots \\
0 & \sin \alpha_{i} & \left(r_{0}-R \cos \alpha_{i}\right) \sin \alpha_{i} & \cdots & 0 & \cdots \\
0 & 0 & -d_{i} \cos \alpha_{i} & \cdots & 1 & \cdots \\
0 & -d_{i} \sin \alpha_{i} & d_{i}\left(R \cos \alpha_{i}-2 r_{0}\right) \sin \alpha_{i} & \cdots & R \sin \alpha_{i} & \cdots \\
0 & d_{i} \cos \alpha_{i} & d_{i}\left(r_{0}-R \cos \alpha_{i}\right) \cos \alpha_{i} & \cdots & \left(r_{0}-R \cos \alpha_{i}\right) & \cdots \\
0 & -r_{0} \sin \alpha_{i} & \left(d_{i}{ }^{2}-r_{0}{ }^{2}+r_{0} R \cos \alpha_{i}\right) \sin \alpha_{i} & \cdots & 0 & \cdots
\end{array}\right)
$$

\section{- Platform}

The hydrostatic force applying on the platform is estimated in a second stage. According to linear theory, one can show that the effect of the Archimedes force over the closed wetted surface $S_{p} \cup S_{i}$ reduces to a constant plus a restoring force proportional to the displacement:

$$
\boldsymbol{F}_{\boldsymbol{H}, \boldsymbol{P} \boldsymbol{i}}\left(G_{0}\right)=\rho g\left(\begin{array}{c}
0 \\
0 \\
V \\
0 \\
-\left(r_{B}-r_{0}\right) V \\
0
\end{array}\right)^{0}-\boldsymbol{K}_{\boldsymbol{P}} \boldsymbol{X}
$$

With $V$ the displaced volume of the platform, and $r_{B}$ the $\mathrm{x}$ coordinate of the buoyancy center in the platform coordinate system.

$\boldsymbol{K}_{\boldsymbol{P}}=\left(\begin{array}{cccccc}0 & 0 & 0 & 0 & \cdots & 0 \\ 0 & 0 & 0 & 0 & \cdots & 0 \\ 0 & K_{P, H 33} & K_{P, H 35} & 0 & \cdots & 0 \\ 0 & 0 & 0 & 0 & \cdots & 0 \\ 0 & K_{P, H 53} & K_{P, H 55} & 0 & \cdots & 0 \\ 0 & 0 & 0 & 0 & \cdots & 0\end{array}\right)$

The hydrostatic contribution from the surface $S_{i}$ was removed in order to obtain the force on the platform alone. From Eq.(13), one can show:

$$
\boldsymbol{F}_{\boldsymbol{H}, \boldsymbol{P}}\left(G_{0}\right)=\rho g\left(\begin{array}{c}
-\sum_{i} d_{i} S_{0} \cos \alpha_{i} \\
-\sum_{i} d_{i} S_{0} \sin \alpha_{i} \\
V \\
\sum_{i} d_{i}^{2} S_{0} \sin \alpha_{i} \\
-\left(r_{B}-r_{0}\right) V-\sum_{i} d_{i}^{2} S_{0} \cos \alpha_{i} \\
-\sum_{i} r_{0} d_{i} S_{0} \cos \alpha_{i}
\end{array}\right)^{0}-\boldsymbol{K}_{\boldsymbol{H}, \boldsymbol{P}} \boldsymbol{X}
$$

With 


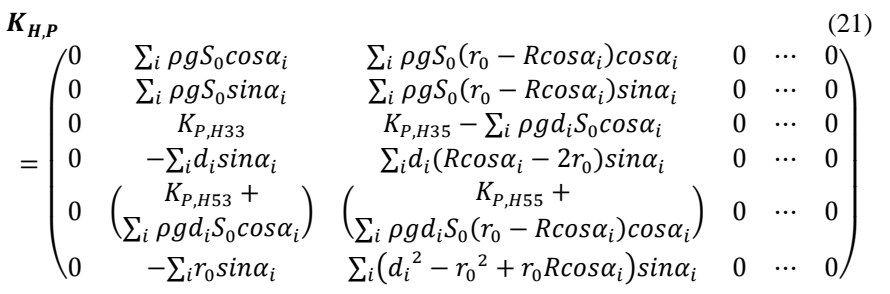

\subsubsection{Aerodynamic force:}

The aerodynamic and gravity forces applying on the wind turbine have an influence on the response of the platform. Let $F_{W T}$ be the thrust on the wind turbine. It can be assumed that it remains oriented along the $\mathrm{x}$-axis, as a first approach. It is resulting from the sum of a static part $F_{W T, 0}$ and a dynamic part proportional to the horizontal velocity of the nacelle. At the nacelle $N\left(x_{N}, 0, z_{N}\right)^{0}$, the horizontal $\cdot$ velocity is $V_{x}(N)=u+$ $z_{N} \dot{\theta_{0}}$. Let $B_{W i}$ be the proportionality coefficient, then:

$$
\overrightarrow{F_{W T}}=\left(\begin{array}{c}
F_{W T, 0} \\
0 \\
0
\end{array}\right)^{0}+B_{W i}\left(\begin{array}{c}
u+z_{N} \theta_{0} \\
0 \\
0
\end{array}\right)^{0}
$$

Thus

$$
\boldsymbol{F}_{W T}\left(G_{0}\right)=\left(\begin{array}{c}
F_{W T, 0} \\
0 \\
0 \\
0 \\
F_{W T, 0} z_{N} \\
0
\end{array}\right)^{0}-\boldsymbol{B}_{W T} \boldsymbol{X}-\boldsymbol{K}_{W T} \boldsymbol{X}
$$

With

$$
\boldsymbol{B}_{W T}=\left(\begin{array}{cccccc}
B_{W i} & 0 & B_{W i} Z_{N} & 0 & \cdots & 0 \\
0 & 0 & 0 & 0 & \cdots & 0 \\
0 & 0 & 0 & 0 & \cdots & 0 \\
0 & 0 & 0 & 0 & \cdots & 0 \\
B_{W i} Z_{N} & 0 & B_{W i} Z_{N}^{2} & 0 & \cdots & 0 \\
0 & 0 & 0 & 0 & \cdots & 0
\end{array}\right)
$$

$\boldsymbol{K}_{W T}=\left(\begin{array}{cccccc}0 & 0 & 0 & 0 & \cdots & 0 \\ 0 & 0 & 0 & 0 & \cdots & 0 \\ 0 & 0 & 0 & 0 & \cdots & 0 \\ 0 & 0 & 0 & 0 & \cdots & 0 \\ 0 & 0 & \left(x_{N}-r_{0}\right) F_{W T, 0} & 0 & \cdots & 0 \\ 0 & 0 & 0 & 0 & \cdots & 0\end{array}\right)$

\subsubsection{Gravity force:}

\section{- WECs}

On the flap $i$, the general gravity force can be written:

$$
\boldsymbol{F}_{\boldsymbol{G}, \boldsymbol{i}}\left(G_{0}\right)=-m_{i} g\left(\begin{array}{c}
0 \\
0 \\
1 \\
R \sin \alpha_{i} \\
\left(r_{0}-R \cos \alpha_{i}\right) \\
0
\end{array}\right)^{0}-\boldsymbol{K}_{\boldsymbol{G}, \boldsymbol{i}} \boldsymbol{X}
$$

With

$$
\boldsymbol{K}_{\boldsymbol{G}, \boldsymbol{i}}=\left(\begin{array}{cccccccc}
0 & 0 & 0 & 0 & \cdots & 0 & \cdots & 0 \\
0 & 0 & 0 & 0 & \cdots & 0 & \cdots & 0 \\
0 & 0 & 0 & 0 & \cdots & 0 & \cdots & 0 \\
0 & 0 & 0 & 0 & \cdots & m_{i} g \sin \alpha_{i} & \cdots & 0 \\
0 & 0 & 0 & 0 & \cdots & -m_{i} g \cos \alpha_{i} & \cdots & 0 \\
0 & 0 & 0 & 0 & \cdots & 0 & \cdots & 0
\end{array}\right)
$$

\section{- Platform}

Knowing that the coordinates of gravity center $G_{W T}$ of the wind turbine are $\left(x_{W T}, 0, z_{W T}\right)^{0}$, the generalized gravity force reads:

$$
\boldsymbol{F}_{G, \boldsymbol{P}}\left(G_{0}\right)=\left(\begin{array}{c}
0 \\
0 \\
-\left(M_{P}+M_{W T}\right) g \\
0 \\
\left(x_{W T}-r_{0}\right) M_{W T} g \\
0
\end{array}\right)^{0}-\boldsymbol{K}_{G, \boldsymbol{P}} \boldsymbol{X}
$$

With

$\boldsymbol{K}_{G, P}=\left(\begin{array}{cccccc}0 & 0 & 0 & 0 & \cdots & 0 \\ 0 & 0 & 0 & 0 & \cdots & 0 \\ 0 & 0 & 0 & 0 & \cdots & 0 \\ 0 & 0 & 0 & 0 & \cdots & 0 \\ 0 & 0 & -z_{W T} M_{W T} g & 0 & \cdots & 0 \\ 0 & 0 & 0 & 0 & \cdots & 0\end{array}\right)$

\subsubsection{PTO force:}

According to section 1.2, the PTO force is modeled as linear and proportional to the relative motion and velocity of the flaps. Therefore, the PTO force applying on each flap can be written

$$
\overrightarrow{F_{P T O, l}}=\overrightarrow{C_{P T O, l}}+\left(-B_{P T O} r_{l}-K_{P T O} r_{i}\right) \overrightarrow{u_{r, l}}
$$

In which the constant $\overrightarrow{C_{P T O, l}}$ aims at cancelling the static parts of the hydrostatic force. $\overrightarrow{u_{r, l}}$ is the normal to the panel $i$. Hence:

$$
\overrightarrow{C_{P T O, \iota}}=\left(\begin{array}{c}
-\rho g d_{i} S_{0} \cos \alpha_{i} \\
-\rho g d_{i} S_{0} \sin \alpha_{i} \\
0
\end{array}\right)^{0}
$$

This force applies at the gravity center of the flap at rest. Therefore, at $G_{0}$, the generalized PTO force reads:

With

$$
F_{P T O, i}\left(G_{0}\right)=C_{P T O, i}-B_{P T O, i} X-K_{P T O, i} X
$$

$\boldsymbol{C}_{\boldsymbol{P T O}, i}=\left(\begin{array}{c}-\rho g d_{i} S_{0} \cos \alpha_{i} \\ -\rho g d_{i} S_{0} \sin \alpha_{i} \\ 0 \\ \rho g d_{i}^{2} S_{0} \sin \alpha_{i} \\ -\rho g d_{i}{ }^{2} S_{0} \cos \alpha_{i} \\ \rho g r_{0} d_{i} S_{0} \sin \alpha_{i}\end{array}\right)^{0}$

$\boldsymbol{B}_{\text {PTO }, \boldsymbol{i}}=B_{P T O}\left(\begin{array}{cccccccc}0 & 0 & 0 & 0 & \cdots & \cos \alpha_{i} & \cdots & 0 \\ 0 & 0 & 0 & 0 & \cdots & \sin \alpha_{i} & \cdots & 0 \\ 0 & 0 & 0 & 0 & \cdots & 0 & \cdots & 0 \\ 0 & 0 & 0 & 0 & \cdots & -d_{i} \sin \alpha_{i} & \cdots & 0 \\ 0 & 0 & 0 & 0 & \cdots & d_{i} \cos \alpha_{i} & \cdots & 0 \\ 0 & 0 & 0 & 0 & \cdots & -r_{0} \sin \alpha_{i} & \cdots & 0\end{array}\right)$

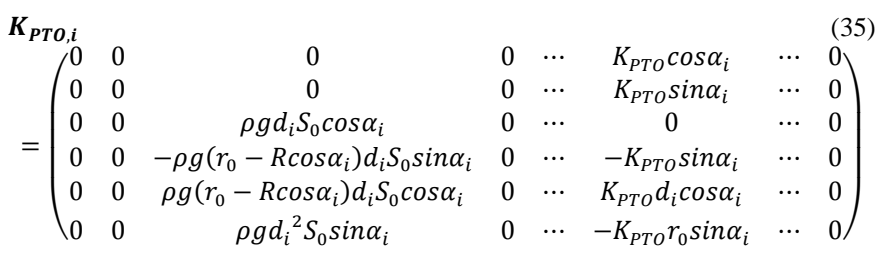


On the platform, the effect of the PTO force is $-\sum_{i} \boldsymbol{F}_{\boldsymbol{P T o}, \boldsymbol{i}}\left(G_{0}\right)$

\subsubsection{Bearing force:}

A bearing force can be modeled to compensate the static part of the gravity force. The bearings are considered perfect and the force applies on $G_{i}$.

$$
\boldsymbol{F}_{\boldsymbol{R}, \boldsymbol{i}}\left(G_{0}\right)=m_{i} g\left(\begin{array}{c}
0 \\
0 \\
1 \\
R \sin \alpha_{i} \\
\left(r_{0}-R \cos \alpha_{i}\right) \\
0
\end{array}\right)^{0}-\boldsymbol{K}_{\boldsymbol{R}, \boldsymbol{i}} \boldsymbol{X}
$$

With

$$
\boldsymbol{K}_{R, i}=\left(\begin{array}{cccccccc}
0 & 0 & 0 & 0 & \cdots & 0 & \cdots & 0 \\
0 & 0 & 0 & 0 & \cdots & 0 & \cdots & 0 \\
0 & 0 & 0 & 0 & \cdots & 0 & \cdots & 0 \\
0 & 0 & 0 & 0 & \cdots & -m_{i} g \sin \alpha_{i} & \cdots & 0 \\
0 & 0 & 0 & 0 & \cdots & m_{i} g \cos \alpha_{i} & \cdots & 0 \\
0 & 0 & 0 & 0 & \cdots & 0 & \cdots & 0
\end{array}\right)
$$

As for the PTO force, the reaction force of the bearings on the platform is $-\sum_{i} \boldsymbol{F}_{\boldsymbol{R}, \boldsymbol{i}}\left(G_{0}\right)$.

\subsubsection{Mooring force:}

As it was demonstrated in [10] and [11], the influence of the mooring layout on the power capture is expected to be small. Hence, it is modeled by a simple restoring force proportional to the horizontal motion of the platform, with a relatively small coefficient.

$$
\boldsymbol{F}_{M, 0}\left(G_{0}\right)=\left(\begin{array}{c}
F_{M} \\
0 \\
0 \\
0 \\
0 \\
0
\end{array}\right)^{0}-\left(\begin{array}{cccccc}
-K_{M} & 0 & 0 & 0 & \cdots & 0 \\
0 & 0 & 0 & 0 & \cdots & 0 \\
0 & 0 & 0 & 0 & \cdots & 0 \\
0 & 0 & 0 & 0 & \cdots & 0 \\
0 & 0 & 0 & 0 & \cdots & 0 \\
0 & 0 & 0 & 0 & \cdots & 0
\end{array}\right) \boldsymbol{X}=\boldsymbol{F}_{\boldsymbol{M}}-\boldsymbol{K}_{\boldsymbol{M}} \boldsymbol{X}
$$

\subsubsection{End-Stop forces:}

The stroke of each flap is limited by end-stops. They are modeled by strong springs which are activated only when the flaps radial motions reach the predefined maximum excursion. The resulting force can be written:

$$
\overrightarrow{F_{e s, l}}=\left(-K_{e s}\left(r_{i}+r_{e s}\right) \mathcal{H}\left(r_{i}+r_{e s}\right)-K_{e s}\left(r_{i}-r_{e s}\right) \mathcal{H}\left(r_{i}-r_{e s}\right)\right) \overrightarrow{u_{r, l}}
$$

In which $K_{e s}$ is the spring coefficient of the end stop and $2 r_{e s}$ is the maximum stroke of the flaps. In Eq.(39), $\mathcal{H}$ is a step function, typically a Heaviside function.

This forces applies on $G_{i}$, thus it creates a momentum in $G_{0}$. The generalized force applied on each flap is $\boldsymbol{F}_{\boldsymbol{e s}, \boldsymbol{i}}\left(G_{0}\right)$ and the reaction on the platform is $-\sum_{i} \boldsymbol{F}_{\boldsymbol{e}, \boldsymbol{i}}\left(G_{0}\right)$.

\subsubsection{Viscous forces:}

It was decided to model the viscous damping force in the time domain only. At the time when this paper was written, the viscous force was being implemented but the results were not fully verified.

\section{4 - Time and Frequency domain equations \\ 2.4.1 Frequency domain:}

In frequency domain (FD), the viscous forces and the endstops are not taken into account. By expressing Newton's law, one can write that the equation of motion for the system \{Platform + Wind Turbine $\}$ :

$$
\begin{gathered}
\boldsymbol{M}_{\boldsymbol{P}} \boldsymbol{X}_{\boldsymbol{P}}=\boldsymbol{F}_{\boldsymbol{e x , \boldsymbol { P }}}-\boldsymbol{C} \boldsymbol{M}_{\boldsymbol{P}} \boldsymbol{T} \boldsymbol{X}-\boldsymbol{C} \boldsymbol{A}_{\boldsymbol{P}} \boldsymbol{T} \boldsymbol{X} \\
-\left(\boldsymbol{K}_{\boldsymbol{H}, \boldsymbol{P}}-\sum_{i} \boldsymbol{K}_{\boldsymbol{H}, \boldsymbol{i}}+\boldsymbol{K}_{\boldsymbol{W T}}+\boldsymbol{K}_{\boldsymbol{G}, \boldsymbol{P}}+\boldsymbol{K}_{\boldsymbol{M}}-\sum_{i} \boldsymbol{K}_{\boldsymbol{P T O}, \boldsymbol{i}}\right) \boldsymbol{X} \\
-\left(\boldsymbol{B}_{W T}-\sum_{i} \boldsymbol{B}_{\boldsymbol{P} T \boldsymbol{O}, \boldsymbol{i}}\right) \boldsymbol{X} \\
+\left(\begin{array}{c}
F_{W T, 0}+F_{M} \\
0 \\
\rho g V-\left(M_{P}+M_{W T}\right) g-\sum_{i} m_{i} g \\
-\sum_{i} m_{i} g R \operatorname{sins} \alpha_{i}-\sum_{i} \rho g d_{i}{ }^{2} S_{0} \sin \alpha_{i} \\
-\rho g\left(r_{B}-r_{0}\right) V+F_{W T, 0} z_{N}+\left(x_{W T}-r_{0}\right) M_{W T} g-\sum_{i} m_{i} g\left(r_{0}-R \cos \alpha_{i}\right) \\
-\sum_{i} \rho g r_{0} d_{i} S_{0} \sin \alpha_{i}
\end{array}\right)
\end{gathered}
$$

At rest, with zero wind speed:

$$
\begin{aligned}
& M_{P}=\rho V-M_{W T}-\sum_{i} m_{i} \\
& F_{M}=-F_{W T, 0} \\
& \left(r_{B}-r_{0}\right)=\frac{1}{\rho V}\left(\sum_{i} m_{i} g\left(r_{0}-R \cos \alpha_{i}\right)+\left(x_{W T}-r_{0}\right) M_{W T}\right) \\
& . .
\end{aligned}
$$

The equation of motion for each flap is:

$$
\begin{aligned}
M_{i} X_{i} & =F_{e x, i}-C M_{i} T X-C A_{i} T X-B_{P T O, i} X \\
& -\left(K_{H, i}+K_{G, i}+K_{P T O, i}\right) X
\end{aligned}
$$

By assembling equations (40) and (44), and multiplying on the left side by $T^{T}$, one gets

With

$$
T^{T}(C M+M) T X=T^{T} F_{e x}-T^{T}\left(C A T+B_{P T O}\right) X-T^{T} K X
$$

$\boldsymbol{M}=\left(\begin{array}{ccc}\boldsymbol{M}_{P} & \cdots & \mathbf{0} \\ \vdots & \ddots & \vdots \\ \mathbf{0} & \cdots & \boldsymbol{M}_{\boldsymbol{i}}\end{array}\right)$

$C M=\left(\begin{array}{c}C M_{P} \\ C M_{i}\end{array}\right)$

$C A=\left(\begin{array}{l}C A_{P} \\ C A_{i}\end{array}\right)$ 
$\boldsymbol{B}_{P T O}=\left(\begin{array}{c}\boldsymbol{B}_{W T}-\sum_{i} \boldsymbol{B}_{P T O, i} \\ \boldsymbol{B}_{P T O, i}\end{array}\right)$

$K=\left(\begin{array}{c}K_{H, P}-\sum_{i} K_{H, i}+K_{W T}+K_{G, P}+K_{M}-\sum_{i} K_{P T O, i} \\ K_{H, i}+K_{G, i}+K_{P T O, i}\end{array}\right)$

\subsubsection{Time domain:}

In the time domain (TD), end stop force forces may be included. The equation of motion reads:

$$
\begin{aligned}
\boldsymbol{T}^{T}\left(\boldsymbol{M}+\boldsymbol{\mu}_{\infty}\right) \boldsymbol{T} \boldsymbol{X}= & \boldsymbol{T}^{T} \boldsymbol{F}_{\text {ex }}-\int_{0}^{t} \boldsymbol{T}^{T} \boldsymbol{K}_{\text {rad }} \boldsymbol{T}(t-\tau) \boldsymbol{X}(\tau) d \tau-\boldsymbol{T}^{\boldsymbol{T}} \boldsymbol{B}_{\boldsymbol{P} T \boldsymbol{O}} \boldsymbol{X} \\
& -\boldsymbol{T}^{\boldsymbol{T}} \boldsymbol{K} \boldsymbol{X}+\boldsymbol{T}^{T} \boldsymbol{F}_{\text {es }}
\end{aligned}
$$

With

$\mu_{\infty}=\lim _{\omega \rightarrow+\infty} C M(\omega)$

$\boldsymbol{K}_{\text {rad }}(t)=\frac{2}{\pi} \int_{0}^{+\infty} \boldsymbol{C A}(\omega) \cos (\omega t) d t$

\section{5 - Implementation}

The BEM code Aquaplus [7] was used to calculate the hydrodynamic coefficients in the frequency domain. Figure 5 displays the mesh used for the calculations. The whole system is discretized with 1260 panels. The hydrodynamic coefficients were calculated at the gravity center of the platform $G_{0}$.
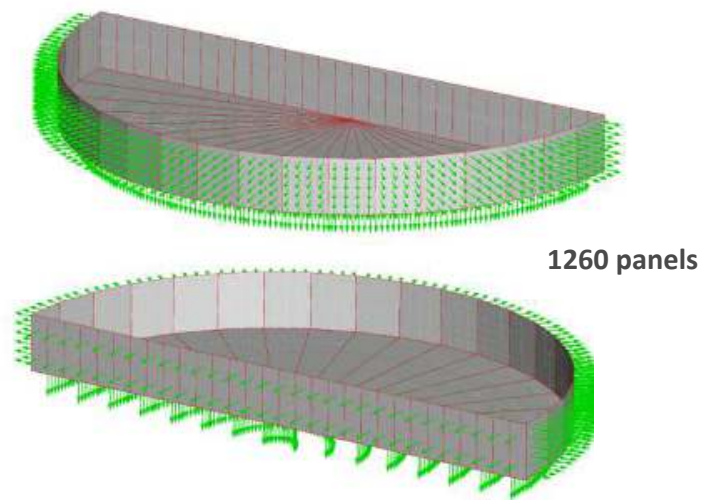

Figure 5: Mesh used for the calculation of the hydrodynamic coefficients.

A refinement check was carried out. In other words, the hydrodynamic coefficients were calculated for a higher number of panels in order to verify the numerical convergence (2120 panels). The agreement was excellent and confirms that the refinement is acceptable.

\section{3- SIMULATION RESULTS AND ENERGY ASSESSMENT}

When they are not explicitly specified, the parameters which were used in all the simulations presented hereafter were provided in Table 1.

\section{1 - Validation tests}

\subsubsection{Pitch response:}

The uncoupled natural pitch period of the system $\{$ platform $+\mathrm{WT}+\mathrm{WECs}\}$ is given by:

$$
\omega_{55,0}=\sqrt{\frac{K_{55}}{I_{55}+C M\left(\omega_{55,0}\right)}}
$$

With $K_{55}$ and $I_{55}$ the hydrostatic stiffness and inertia of the locked system. In fact, the pitch period can easily be calculated provided that the flaps are locked. Setting the PTO parameters significantly high allows to keep the flaps in place. After a few iterations, one gets $\omega_{55,0}=0.74 \mathrm{rad} / \mathrm{s}$.

Figure 6 shows the RAO (Response Amplitude Operator) in pitch with high PTO forces for each flap. Two different peaks can be observed. The lower frequency resonance depends on the interaction between surge and pitch motion due to the mooring forces. The second peak corresponds to the natural pitch period. A very good agreement between the formula and the FD model appears.

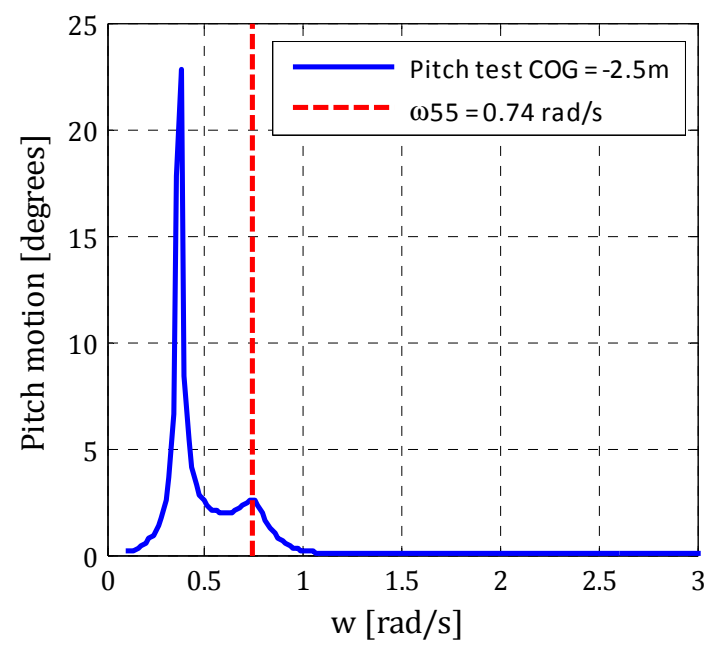

Figure 6: RAO of the Marina Platform computed with the frequency domain (FD) model.

Such a resonance around 8 s could be seen as a stability issue because the main wave period in the Atlantic is very close to $9 \mathrm{~s}$. Different positions of $G_{0}$ were tested without any significant improvement of the natural period. In fact, the substantial waterplane area of this design induces a large second moment of area, which leads to a high pitch stiffness. A change of shape, with perhaps a hollow D-shape, would enable to reduce the pitch natural frequency. A detailed design phase will take place in the MARINA project at a later stage.

At the time of writing this paper, it was decided to assess the performance of this device in the ideal case where the platform is fixed. Indeed, if the power results are not satisfying for such an idealistic case, the focus should be directed to another type of platform. 


\subsubsection{FD and TD comparison:}

The equation of motion was solved using both FD and TD models, and the results are displayed for two of the twenty flaps. As it was described in 2.3.1, panel4 is located on the side of the platform whereas panell3 is facing the incoming waves. Figure 7 and Figure 8 present the results of this comparison for the radial motion and the power outputs of each flap. The wave amplitude was set to $1 \mathrm{~m}$, the platform is fixed (see 3.1.1), and the PTO settings were $[B P T O=5 e 5 \mathrm{Ns} / \mathrm{m} ; \mathrm{KPTO}=5 \mathrm{e} 5 \mathrm{~N} / \mathrm{m}]$.

One can see that the agreement is excellent for most frequencies, except for a narrow range around $1.1 \mathrm{rad} / \mathrm{s}$. The main reasons are still unclear. It could be related to a trapped mode phenomenon. Indeed, for infinite water depth, this frequency corresponds to a wavelength of $50 \mathrm{~m}$. The radius of the platform being exactly $50 \mathrm{~m}$, it seemed realistic to relate this issue with radiation trapped modes.

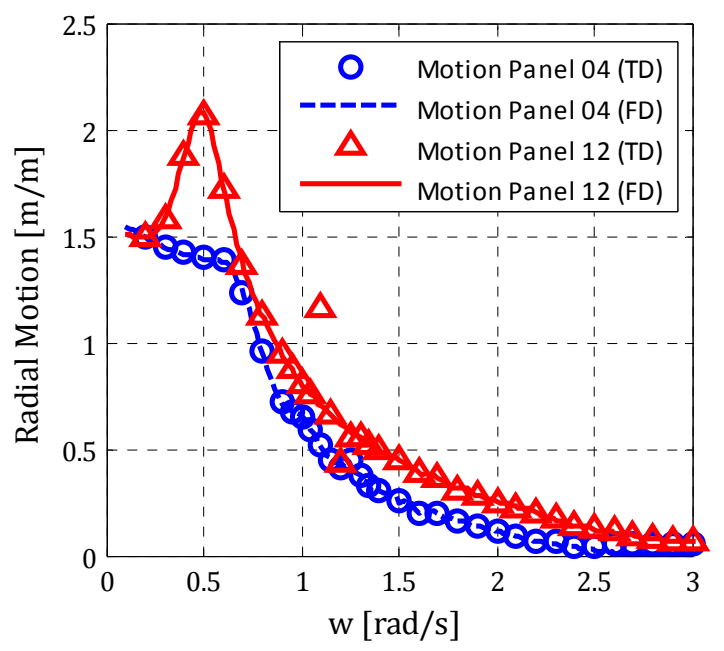

Figure 7: Comparison of the Panels' RAOs computed with the FD and TD models. The viscous forces are set to zero.

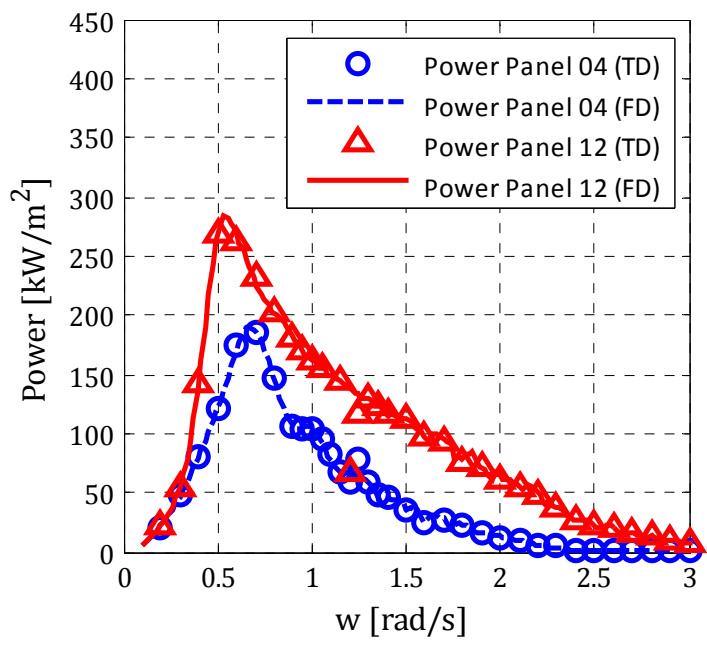

Figure 8: Comparison of the Panels' power outputs computed with the FD and TD models. The viscous forces are set to zero.
Nevertheless, Figure 7 and Figure 8 show that the implementations of the equation of motions are correct in the two models, at least for the first order. In addition, most of the results presented below were obtained with the FD model, which does not show any irregularity.

\subsubsection{End stop test:}

Finally, a verification of the implementation of the endstops in the TD model was carried out. The motion response of each flap was computed for a 10seconds period and 3meters height regular wave. The maximum excursion of each flap was limited to $4 \mathrm{~m}$, which corresponds to a $8 \mathrm{~m}$ stroke limit.

Figure 9 displays the results for two flaps, one on the side and the other one from the front of the platform. The behavior observed was expected.

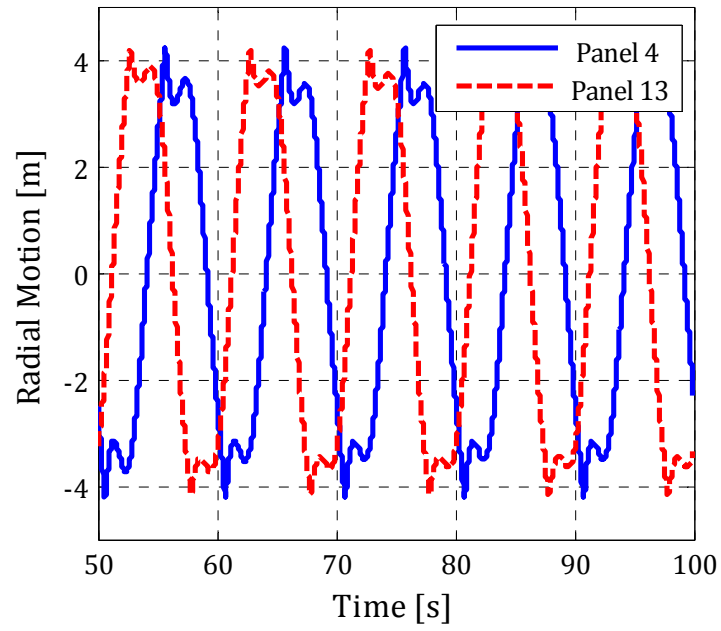

Figure 9: Motion response of panels 4 and 13, with a stroke limit set to $4 \mathrm{~m}$.

\section{2 - Initial PTO assessment}

The PTO settings have an influence on the flaps motion, as well as on the power output. In order to understand the influence of the PTO parameters, the equation of motion was solved with several sets of PTO coefficients. The force is assumed to be linear as described in 2.3.5, and the different settings are presented in Table 2.

\begin{tabular}{cccc}
\hline Settings & $\begin{array}{c}\mathbf{B}_{\text {PTO }} \\
{[\mathrm{kNs} / \mathrm{m}]}\end{array}$ & $\begin{array}{c}\mathrm{K}_{\text {PTO }} \\
{[\mathrm{kN} / \mathrm{m}]}\end{array}$ \\
$\mathbf{1}$ & 100 & 200 \\
$\mathbf{2}$ & 100 & 400 \\
$\mathbf{3}$ & 100 & 600 \\
$\mathbf{4}$ & 500 & 200 \\
$\mathbf{5}$ & 500 & 400 \\
$\mathbf{6}$ & 500 & 600 \\
$\mathbf{7}$ & 1000 & 200 \\
$\mathbf{8}$ & 1000 & 400 \\
$\mathbf{9}$ & 1000 & 600 \\
\hline
\end{tabular}

Table 2: PTO settings for the linear damper. 

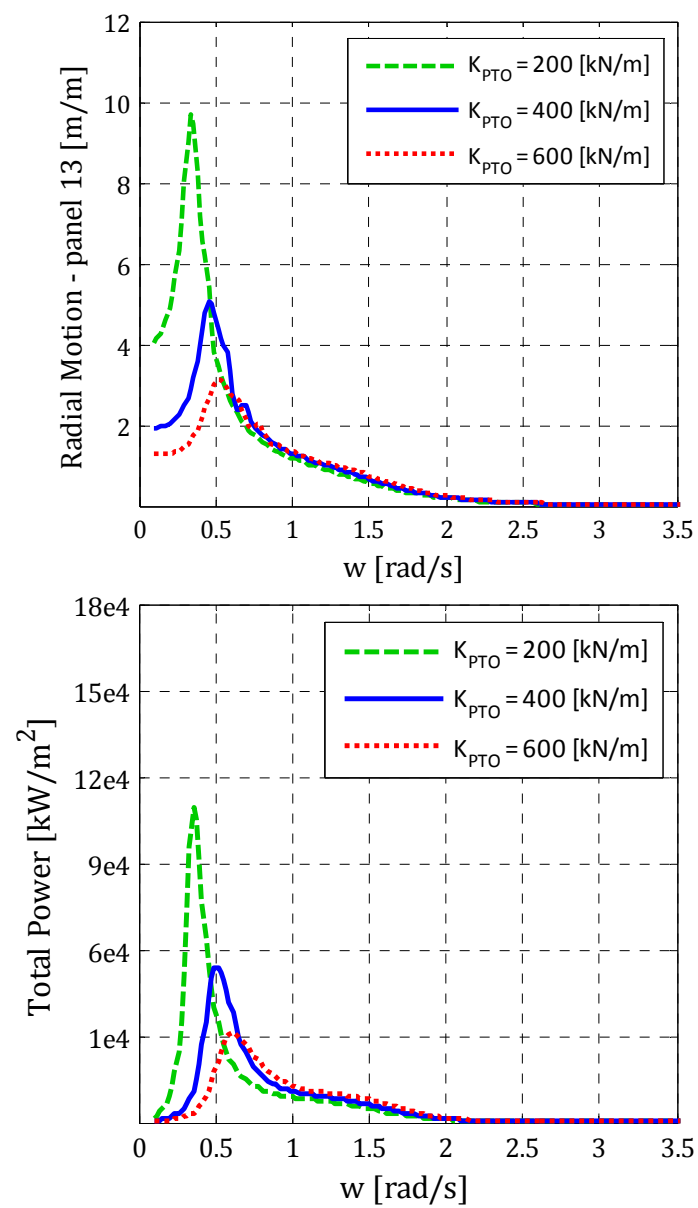

Figure 10: Motion response for panel 13, and total power output for setting 1 to 3 .

The two graphs in Figure 10 show motion and power results for settings 1 to 3 , knowing that the PTO damping is low. The main outcomes are:

- The total power absorbed by the platform can be as high as $100 \mathrm{MW}$ per square meter of incident wave. However, it corresponds to unrealistically large flap excursions (up to $10 \mathrm{~m}$ ). It is likely to be damped by non-linear viscous effect.

- As expected, a change in the PTO stiffness gives the possibility to tune the natural period of the system.

On the other hand, Figure 11 presents the outputs for settings 2, 5 and 8, with varying $B_{P T O}$ values and for a given setting of the stiffness $\left(K_{P T O}=400 \mathrm{kN} / \mathrm{m}\right)$. It can be observed that:

- The amplitude of the motion is reduced as the PTO damping increases.

- The power function also strongly depends in this parameter. When $B_{\text {PTO }}$ reaches very high values, the motion is over-damped and the power decreases. An optimal has to be searched for each sea state, in order to improve the power capture.
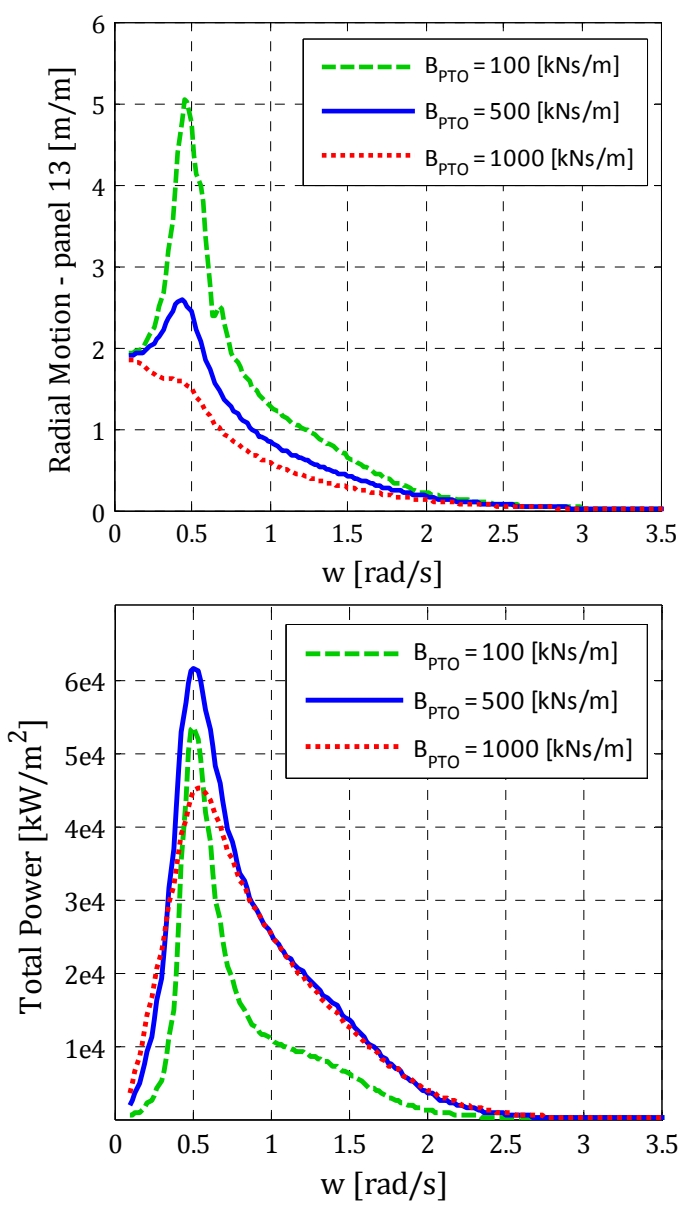

Figure 11:Motion response for panel 13, and total power output for setting 2,5 and 8 .

\section{3 - Power Matrix and specifics}

\subsubsection{Power Matrix:}

In this section, all the frequency domain simulations were performed in irregular waves using the JONSWAP spectrum. At the time when the paper was written, time domain results were not yet available. For each sea state, the PTO settings $\left(B_{P T O}\right.$, $K_{\text {PтO) }}$ ) were selected from a given range of values (see Table 3 ). This methodology is the brute force approach of a PTO optimization.

\begin{tabular}{l|c|c} 
Settings & B $_{\text {PTO }}[\mathrm{kNs} / \mathrm{m}]$ & $\mathrm{K}_{\text {PTO }}[\mathrm{kN} / \mathrm{m}]$ \\
Minimum & 100 & 100 \\
Maximum & 1000 & 1000
\end{tabular}

Table 3: Range of PTO settings.

As it was expected from linear theory, the optimal PTO coefficients are independent from the characteristic wave height. The maximum PTO damping value selected is around $600 \mathrm{kNs} / \mathrm{m}$ for peak periods close to $7 \mathrm{~s}$. As far as the stiffness coefficient is concerned, it becomes smaller when the peak period increases. It would actually tend towards negative values if the range was not restricted to positive values (see 1.2). 


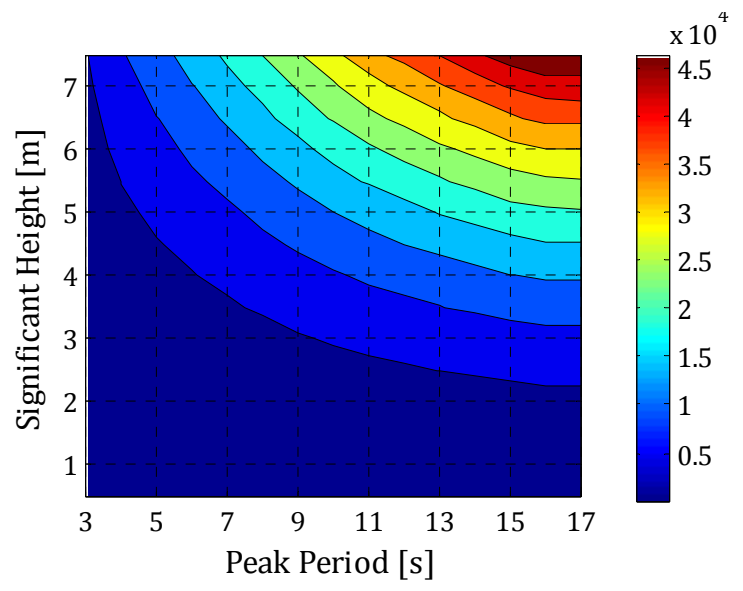

Figure 12: Power matrix for the platform considered.

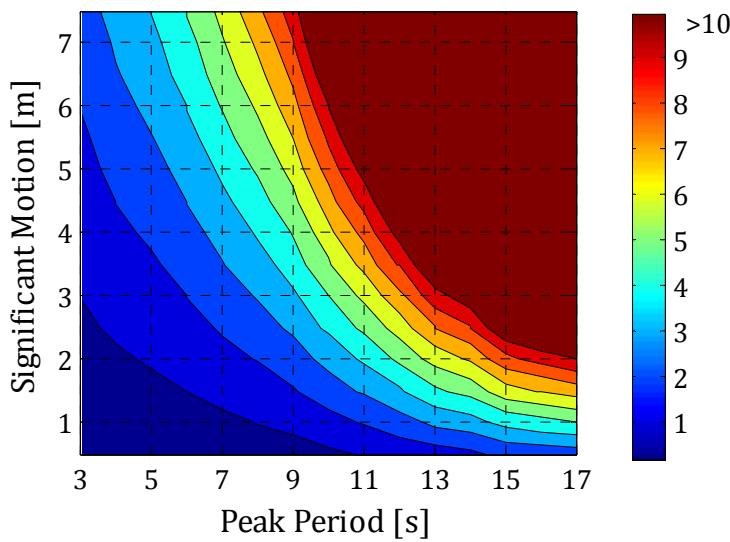

Figure 13: Significant motion for the optimized PTO settings.

Figure 12 shows the power matrix of the platform considered, which is independent of the geographical site. The very high values observed on the top right corner represent the power absorbed for very rare and energetic sea states. As only frequency domain results are available, the influence of the PTO settings on the platform motion has been analyzed based on the RMS value for the flaps motion (see Figure 13).

By multiplying the power matrix with wave data statistics, one can calculate the annual energy absorption for different wave sites. The values are reported in Table 4 as well as the cost indicators defined in [4].

\begin{tabular}{|l|l|cc|ccc|}
\hline Site & Units & Yeu & EMEC & SEM-REV & Lisboa & Belmullet \\
\hline $\boldsymbol{V}$ & & 3.3 & 1 & 1 & 3.3 & 3.3 \\
\hline Mean Power & {$[\mathrm{MW}]$} & 3.3 & 2.6 & 1.7 & 4.7 & 10.2 \\
\hline Wave resource & {$[\mathrm{kW} / \mathrm{m}]$} & 26.2 & 21.4 & 14.8 & 36.8 & 79.1 \\
\hline Capture width & {$[\%]$} & 126 & 120 & 118 & 127 & 129 \\
\hline Energy / $\mathbf{V}$ & {$\left[\mathrm{MWh} / \mathrm{m}^{3}\right]$} & 0.74 & 0.58 & 0.39 & 1.05 & 2.29 \\
\hline Energy / $\mathbf{S}_{\text {tot }}$ & {$\left[\mathrm{MWh} / \mathrm{m}^{2}\right]$} & 2.46 & 1.92 & 1.31 & 3.51 & 7.62 \\
\hline
\end{tabular}

Table 4: MARINA Platform power criteria.
The main outcomes from Table 4 are:

- The annual power figures obtained are higher than expected. Indeed, a simple capture width ratio calculation (based on $100 \mathrm{~m}$ characteristic length and a typical surge WEC ratio around $41 \%$ ) would suggest an annual result around 1MW for the Yeu site. Using the semi-perimeter $(157 \mathrm{~m})$, as the characteristic length, leads to a final result around 1.7MW. Nevertheless, even if results are high, the order of magnitude appears to be correct.

- The cost indicators such as the Energy/Displacement or the Energy/Surface is closer to results published in [4].

- The very high power results of this platform can be explained by the fact that the energy lost by radiation is low. Indeed, the flaps can radiate wave only from their front side, which is similar to an ideal wave-maker more than a typical point absorber device.

- For a typical sea state $(\mathrm{Tp}=10 \mathrm{~s} ; \mathrm{Hs}=2.5 \mathrm{~m})$, the significant excursion of a flap is $3.5 \mathrm{~m}$. It seems reasonable for a $15 \mathrm{~m}$ long panel.

\subsubsection{Power smoothing:}

One of the initial arguments in favor of this platform was the capacity to produce a smooth power output. Indeed, knowing that each flap will absorb power with a different phase, the electrical power output is likely to be smoother. Such a characteristic can significantly reduce the installation cost with a lower power cable rating.

A simple test was carried out with the TD model, without viscous forces or end-stops reaction. For regular and irregular waves an interesting smoothing phenomenon was observed on the total power. It was decided to show the results for an irregular test mainly because it represents a more realistic situation. Therefore, Figure 14 displays the time dependent power output for three of the twenty flaps, together with the total power resulting from the contribution of all flaps. The wave computed had a 10seconds peak period and a significant height of 2.5 meters. The normalized standard deviation for the total power signal is about 0.9 whereas it is closer to 1.3 for the three different panels. These statistical considerations can confirm the trend observed on the time traces. The final power output will be smoother than for usual point absorbers, which can represent a significant cost advantage. 

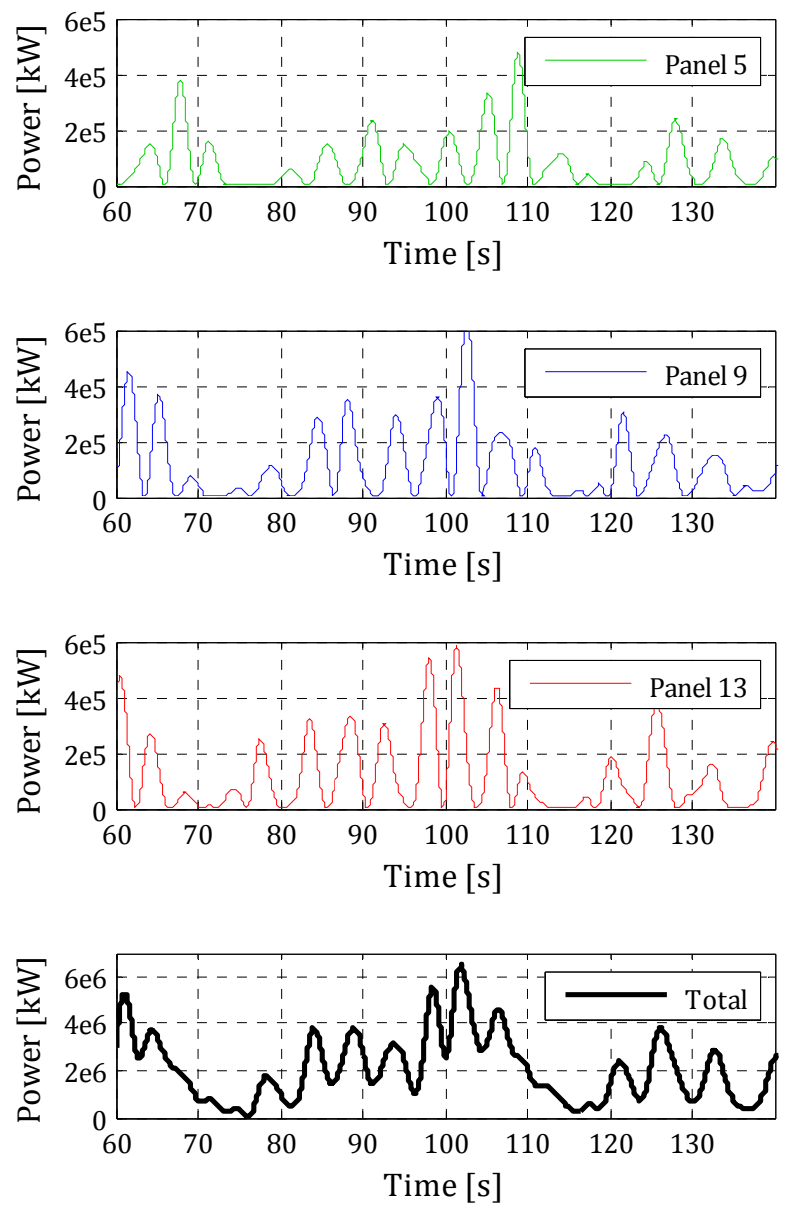

Figure 14: Different panel power outputs together with the total power created by all the WECs on the platform.

\section{4- CONCLUSION AND FURTHER DEVELOPMENT}

The main outcomes from this study have been described in the results shown above, and are summarized below:

- The mean power level that one can expect in the ideal case of a fixed platform is about $3 \mathrm{MW}$ in a site where the resource is about $25 \mathrm{~kW} / \mathrm{m}$.

- The output power from such a device is $30 \%$ smoother that for usual WECs based on 1 degree of freedom power absorption.

- For this particular hybrid platform, the annual energy production from a typical $5 \mathrm{MW}$ wind turbine seems to be balanced by the WECs.

- The very good initial power results are encouraging and justify the interest and the simulation work related to this concept.

The time domain model is being upgraded with non-linear viscous forces together with a control system aiming at improving the stability of the platform. Additional results from time domain will certainly provide more details concerning this platform. A second design phase will intend to improve the device performance and to increase the overall stability. The MARINA project will run until 2014, thus, further work will be carried out in order to confirm the conclusions presented in this paper, and to clarify the uncertainties inherent to the numerical modelling assumptions. Furthermore, the project identified the need for tank testing the most promising devices. Providing that future numerical simulations do not create any show stopper, the platform presented in this paper could be tested in a wave basin.

\section{ACKNOWLEDGMENTS}

The authors gratefully acknowledge the financial support from the European Commission through the $7^{\text {th }}$ Framework Programme (MARINA Platform - Marine Renewable Integrated Application Platform, Grant Agreement 241402) which made this work possible.

\section{REFERENCES}

[1] http://www.marina-platform.info

[2] A. Babarit, J. Hals, 2011. On the maximum and actual capture width ratio of wave energy converters. Proc of the $9^{\text {th }}$ EWTEC conference.

[3] N.W.Bellamy, 1985.The Sea Clam Wave Energy Converter. Proc. of the ASME Third International Offshore Mechanics and Arctic Engineering

[4] A. Babarit, J. Hals, M.J. Muliawan, A. Kurniawan, T. Moan, J. Krokstad, 2012. Numerical benchmarking study of a selection of wave energy converters. Renewable Energy, Vol. 41, pages 44-63.

[6] J. Jonkman, S. Butterfield, W. Musial, G. Scott, 2009. Definition of a 5-MW Reference Wind turbine for Offshore System Development. National Renewable energy Laboratory.

[7] G. Delhommeau, 1997. Seakeeping Code Aquaplus.

[8] J. Falnes, 2002. Ocean Wave and oscillating systems. Linear interactions including wave energy extraction. Cambridge university press.

[9] B. Molin, 2002. Hydrodynamique des structures offshore, Guides Pratiques sur les Ouvrages en Mer, TECHNIP Eds.

[10] M.J. Muliawan, Z. Gao, T. Moan, A. Babarit, 2011. Analysis of a two-body floating wave energy converter with particular focus on the effect of mooring system on energy capture. Proc. of International Conference on Ocean, Offshore and Artic Engineering.

[11] P.C.Vicente, A.F. de O. Falcão, L.M.C. Gato, P.A.P. Justino, 2009. Dynamics of arrays of floating point-absorber wave energy converters with inter-body and bottom slackmooring connections, Applied Ocean Research, Vol. 31 (4), pages 267-281. 